\title{
Transcriptome assembly for a colour- polymorphic grasshopper (Gomphocerus sibiricus) with a very large genome size
}

\author{
Abhijeet Shah ${ }^{1,2^{*}}$ (D) Joseph I. Hoffman² and Holger Schielzeth ${ }^{1}$
}

\begin{abstract}
Background: The club-legged grasshopper Gomphocerus sibiricus is a Gomphocerinae grasshopper with a promising future as model species for studying the maintenance of colour-polymorphism, the genetics of sexual ornamentation and genome size evolution. However, limited molecular resources are available for this species. Here, we present a de novo transcriptome assembly as reference resource for gene expression studies. We used highthroughput Illumina sequencing to generate 5,070,036 paired-end reads after quality filtering. We then combined the best-assembled contigs from three different de novo transcriptome assemblers (Trinity, SOAPdenovo-trans and Oases/Nelvet) into a single assembly.

Results: This resulted in 82,251 contigs with a N50 of 1357 and a TransRate assembly score of 0.325, which compares favourably with other orthopteran transcriptome assemblies. Around $87 \%$ of the transcripts could be annotated using InterProScan 5, BLASTx and the dammit! annotation pipeline. We identified a number of genes involved in pigmentation and green pigment metabolism pathways. Furthermore, we identified 76,221 putative single nucleotide polymorphisms residing in 8400 contigs. We also assembled the mitochondrial genome and investigated levels of sequence divergence with other species from the genus Gomphocerus. Finally, we detected and assembled Wolbachia sequences, which revealed close sequence similarity to the strain pel wPip.

Conclusions: Our study has generated a significant resource for uncovering genotype-phenotype associations in a species with an extraordinarily large genome, while also providing mitochondrial and Wolbachia sequences that will be useful for comparative studies.
\end{abstract}

Keywords: Insects, Orthoptera, Acrididae, Gomphocerinae, Transcriptome, Mitochondria, Wolbachia

\section{Background}

One important goal of functional genomics is to establish links between genetic polymorphisms and phenotypic variation [1]. Recent developments in high-throughput sequencing technologies have greatly facilitate this endeavour. However, there are still major challenges inherent to genomic approaches for genotype-phenotype associations in non-model organisms [2]. One of these challenges is imposed by species with large genomes, as genome assemblies are difficult to construct for highly repetitive regions [3]. To some degree, this issue will be mitigated by the

\footnotetext{
* Correspondence: abhijeet.shah@uni-jena.de

${ }^{1}$ Institute of Ecology and Evolution, Friedrich Schiller University Jena, Dornburger Str. 159, 07743 Jena, Germany

${ }^{2}$ Department of Animal Behaviour, Bielefeld University, Morgenbreede 45, 33615 Bielefeld, Germany
}

development of long-range sequencing technologies [4]. Nevertheless, we expect that genomic approaches for taxa with very large genomes will remain challenging for some time.

Transcriptomics offers an alternative to genomic approaches, as the size of the transcriptome does not scale linearly with genome size and most functional differences with phenotypic effects should be reflected in the transcriptome [5]. Not only do transcripts differ in their sequences, mirroring underlying coding DNA sequence differences, but quantitative analysis can also allow the assessment of regulatory variation influencing transcript abundance [6, 7]. One prerequisite for an analysis of transcript abundance is a high quality transcriptome assembly, as this acts as a reference against which short read RNA sequencing data can be mapped [8].

(c) The Author(s). 2019 Open Access This article is distributed under the terms of the Creative Commons Attribution 4.0 International License (http://creativecommons.org/licenses/by/4.0/), which permits unrestricted use, distribution, and reproduction in any medium, provided you give appropriate credit to the original author(s) and the source, provide a link to the Creative Commons license, and indicate if changes were made. The Creative Commons Public Domain Dedication waiver (http://creativecommons.org/publicdomain/zero/1.0/) applies to the data made available in this article, unless otherwise stated. 
Grasshoppers from the family Acrididae (Orthoptera, Caelifera) encompass a large number of species, including several economically relevant species, but have been poorly studied molecularly, partly because of their often very large genomes [9]. The 1Kite project has set out to sequence 1000 insect transcriptomes, including 43 species of Orthoptera, but only two Acridids (http://1kite.org/ downloads/1KITE_species.txt). This is surprising because the Acridids are the largest family of Orthopterans, comprising around half of all Orthopteran species [10]. The only Acridid transcriptomes published so far are for the desert locust Schistocerca gregaria [11], the migratory locus Locusta migratoria [12], the stripe-winged grasshopper Stenobothrus lineatus [13] and the bow-winged grasshopper Chorthippus biguttulus [14].

The two locusts, Schistocera and Locusta, belong to the subfamilies Cyrtacanthacridinae and Oedipodinae respectively and have moderately large genomes [9]. Both species are of great interest due to their involvement in pest outbreaks and remarkable phenotypic plasticity. However, true phase-polymorphism and swarming behaviour is rather unusual, even among Acridids [15]. From an evolutionary perspective, the Acridids are remarkable for another phenomenon, the taxonomically widespread occurrence of an apparently balanced green-brown polymorphism in body coloration [16, 17].

The genera Stenobothrus and Chorthippus are representatives of the Gomphocerinae, a large subfamily of Acididae with particularly large genomes [9] and widespread green-brown polymorphisms, but no locust-like swarming behaviour. Of those two, only Chorthippus biguttulus is green-brown polymorphic.

Here, we present a de novo transcriptome assembly for the club-legged grasshopper Gomphocerus sibiricus, an alpine-dwelling species that is unusual for its striking sexual dimorphism in front leg morphology [18] (see Fig. 1) and is also characterized by the widespread occurrence of a balanced green-brown polymorphism [17]. The green-brown polymorphism has been found to be unrelated to rearing conditions [19] and recent evidence from the laboratory suggests that it follows a simple Mendelian mode of inheritance (unpublished data). Biliverdin is the dominant green pigment in Orthopterans [20] and it is likely that the biliverdin synthesis pathway plays an important role in the determination of body colour. However, the specific genes involved in producing the green-brown polymorphism are unknown in this species as well as in grasshoppers in general. Transcriptomic analysis thus offers a promising route for uncovering the genetic basis of this eco-evolutionarily relevant trait in club-legged grasshoppers as well as potentially in other species.

We assembled cDNA sequencing data from five $G$. sibiricus individuals including both males and females as

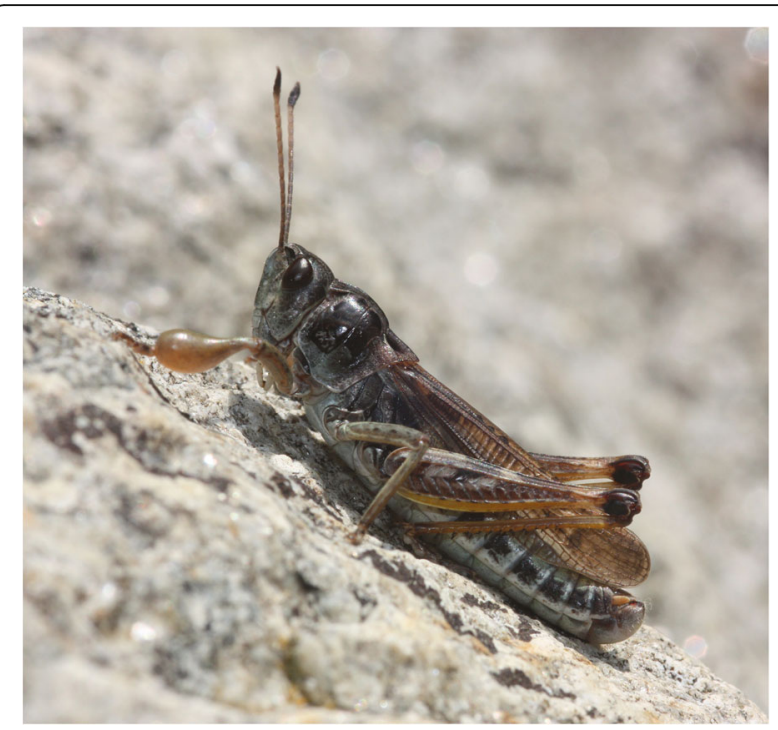

Fig. 1 The club-legged grasshopper Gomphocerus sibiricus, an alpine-dwelling species exhibiting prominent sexual dimorphism of the front leg. Photo credit: Holger Schielzeth

well as specimens of the two colour morphs. In order to generate the most exhaustive assembly possible, we employed three different commonly used assemblers and combined the resulting assemblies into a single high-quality transcriptome assembly [21, 22]. We then compared our assembly to other published grasshopper assemblies and mined the transcripts for the presence of candidate genes involved in biliverdin pigmentation pathways. Additionally, we analysed the expression profile of the mitochondria and compared this to data from closely related species. Finally, having also found evidence for the presence of the parasitic microbe Wolbachia in our sample, we decided to assemble and analyse the Wolbachia strain present in this grasshopper species.

\section{Results}

Transcriptome assembly assessment and completeness metrics

We assembled a draft G. sibiricus transcriptome using three assemblers, followed by pooling of assemblies and removal of duplicates. The final draft assembly comprised 82,251 contigs, 21,347 of which contained open reading frames (ORFs). The TransRate transcriptome assembly metric was 0.325 , which is similar to or better than most previously published orthopteran transcriptomes (Table 1). In order to further assess the quality of the transcriptome assembly, we constructed a kernel density plot of contig lengths versus the average depth of sequences mapping to those contigs (Additional file 1: Figure S1). Overall, the mapping rate using both read datasets was $96.72 \%$ (97.02\% when using normalised reads, read mapping statistics for non-rRNA reads only 
Table 1 TransRate assembly metrics and BUSCO completeness assessment for Gomphocerus sibiricus (this study) in comparison to the other two Acridid transcriptomes published (Stenobothrus lineatus, [13], Chorthippus biguttulus, [34]). Higher TransRate assembly scores indicate better quality assemblies. BUSCO completeness assessment was conducted using the insect ortholog database (orthoDB9)

\begin{tabular}{llll}
\hline TransRate metrics & Gomphocerus sibirucus & Stenobothrus lineatus & Chorthippus bigullutus \\
\hline Number of contigs & 82,251 & 57,778 & 67,733 \\
Number of contigs with ORF & 21,347 & 12,717 & 30,018 \\
N50 of contig length & 1357 & 1207 & 1246 \\
Length of longest contig & 43,026 & 22,561 & 34,437 \\
Length of shortest contig & 301 & 200 & 600 \\
Proportion of read fragments mapped & 0.88 & 0.70 & 0.51 \\
Proportion of good read pairs mapping & 0.82 & 0.63 & 0.43 \\
TransRate assembly score & 0.325 & 0.162 & 0.106 \\
BUSCOs (Number of BUSCO units found) & & & 1489 \\
$\quad$ Complete BUSCOs & 1405 & 1337 & 1323 \\
Complete and Single-Copy BUSCOs & 1093 & 1244 & 166 \\
Complete and duplicated BUSCOs & 312 & 93 & 99 \\
$\quad$ Fragmented BUSCOs & 137 & 142 & 179 \\
$\quad$ Missing BUSCOs & 116 & 1658 & 1658 \\
Total BUSCOs searched & 1658 & & 70 \\
\hline
\end{tabular}

are available in Additional file 7: Table S3) with BWA under default settings. The mean (median) contig length was $1057 \mathrm{bp}(718 \mathrm{bp})$ and the mean (median) coverage was $52.6 \mathrm{x}(11 \mathrm{x})$, indicating that we were able to assemble relatively complete contigs.

\section{Transcriptome annotation and GO classification}

Over $87 \%$ of the contigs were annotated using InterProScan 5, BLASTx (non-redundant protein database) and the dammit! annotation pipeline. The InterProScan analysis resulted in 1,588,733 matches from 72,132 annotated sequences, with 337,258 assigned membrane-bound protein signatures. Furthermore, 109,437 GO terms were assigned to 39,794 transcripts. A BLASTX search of transcripts was run against the non-redundant (nr) protein database which yielded 12,436,946 hits from 31,527 transcripts. Around $78 \%(24,568)$ of these transcripts were assigned to insects, and the next largest fraction (3.8\%) was assigned to the Arachnida (Additional file 2: Figure S2). The dammit! annotation pipeline provided us with 363,218 annotations from 44,488 transcripts. Of these, 41,535 transcripts were annotated by both the InterProScan 5 and dammit! Pipelines (Additional file 5: Table S1).

The annotated draft transcriptome was classified into three main categories of GO components: cellular components, molecular function and biological processes (Additional file 3: Figure S3). Of these, 3617 (9.1\%) were classified as cellular component, 26,974 (67.8\%) as molecular function and $9203(23.2 \%)$ as biological process.
Furthermore, GO terms associated with accumulation of pigment and haeme biosynthesis (GO:0043473 pigmentation; GO:0006783, GO:0006784 haeme; GO:0048034 haeme-O and haeme oxygenase decyclizing activity) were found amongst these annotations. Additionally, we found several relevant InterPro terms: IPR015118 (5-aminolevulinate synthase presequence), IPR010961 (tetrapyrrole biosynthesis of 5-aminovulenic acid), IPR002051 (haeme oxygenase) and KEGG reference: $00860+1.14 .14 .18$ (biliverdin producing haeme oxygenase) in our annotations. This provides a starting point for investigating differential gene expression in relation to the green-brown colour polymorphism in future studies.

\section{SNP calling and estimation of minor all frequencies (MAF)} We found 76,221 SNPs in 84,00 contigs with mean of 9.07 variants per contig. A contour plot shows that minor allele frequencies (MAFs) peak between 0.12 and 0.2 , while rarer variants could only be detected with increasing depth of sequencing coverage (Fig. 2).

\section{Scanning for expressed mitochondrial genes in assembled transcripts}

G. sibiricus mitochondrial sequences were also found in the assembled transcripts, and their coverage depth was estimated. An alignment of the assembled draft transcripts against the published mitochondrial genome [23] shows that we could recover large 


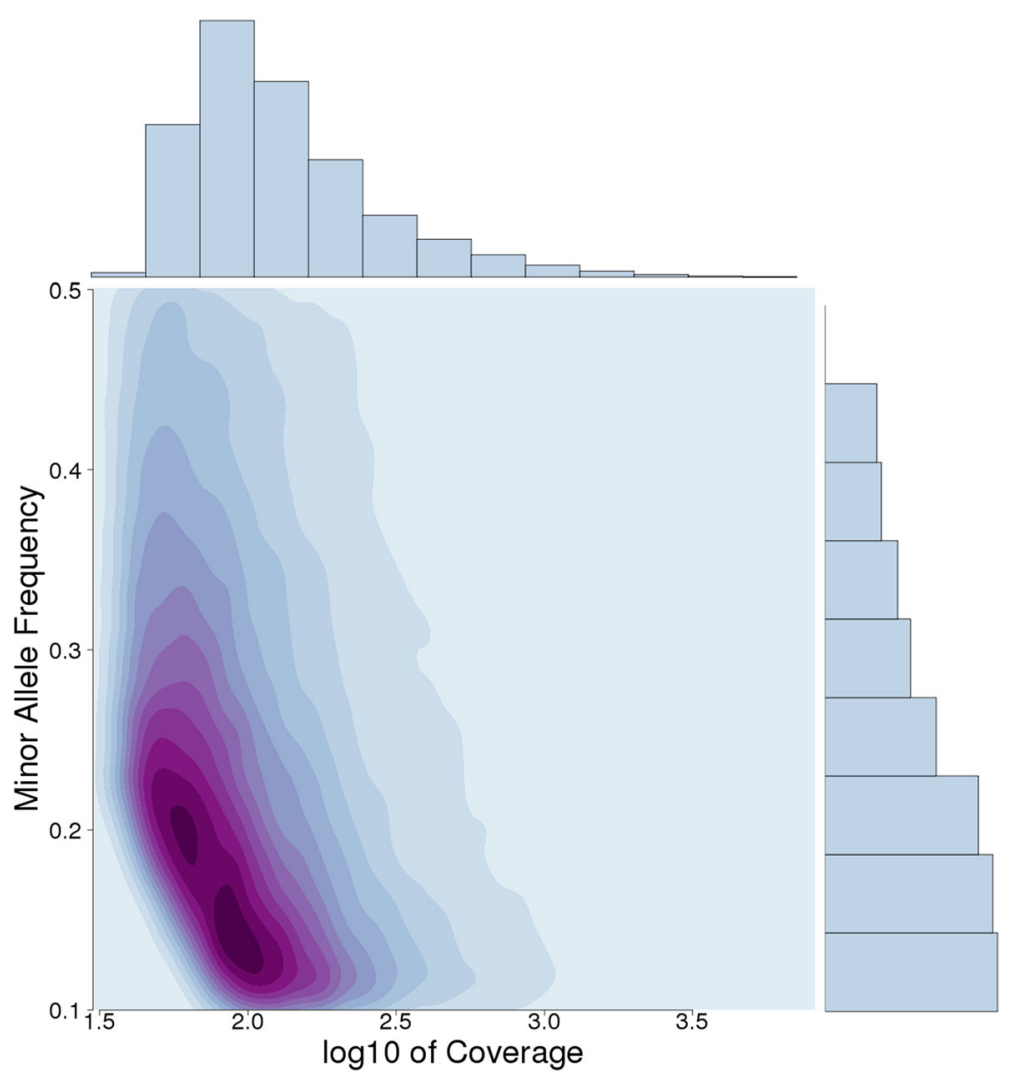

Fig. 2 A two-dimensional kernel density plot showing minor allele frequency (MAF) plotted against log 10 sequence coverage. The dark purple regions indicate higher densities, whereas the light blue regions indicate lower densities. Marginal histograms show frequency distributions of minor allele frequency (top axis) and coverage (right axis)

multi-gene segments. The published mitochondrial genome of G. sibiricus (refseq: NC_021103.1) consists of a standard set of 13 protein coding genes and two ribosomal RNA genes $(16 \mathrm{~s}$ and $12 \mathrm{~s}$ ribosomal sub-units). As expected, our contigs mapped to the reference across the full mitochondrial genome with particularly high coverage over the $16 \mathrm{~s}$ and $12 \mathrm{~s}$ ribosomal RNA genes (Fig. 3).

The distance matrix of the mitochondrial genome assembled from this study and four closely related taxa provides an overview of the amount of sequence divergence within the genus Gomphocerus. As expected, the lowest divergence was found between our European G. sibiricus population and the Asian population of the same species (divergence 1.6\%, Additional file 6: Table S2), while there was slightly more divergence with the Asian congeneric species G. licenti and G. tibetanus, and the largest divergence was found in the comparison with G. rufus (which is usually placed within the genus Gomphocerippus). This analysis also uncovered two unexpected patterns. First, G. sibiricus was found to be less divergent from licenti than from tibetanus, even though the latter is sometimes considered a subspecies of $G$. sibiricus [10]. Second divergence estimates with licenti / tibetanus were lower than with the published G. sibiricus sequence from Central Asia.

\section{Detection of Wolbachia Pel wPip strain sequences}

We detected the endosymbiont Wolbachia in our RNA sequencing data. For strain determination, we mapped our Wolbachia assembly to all the Wolbachia genomes available on GenBank (8280 assemblies, retrieved 30/11/ 2017) using $B W A$ aligner. Our Wolbachia strain showed the best mapping to strain wPip, which was originally described from the Culex quinquefasciatus Pel genome [24]. Mean read coverage was quantified using a sliding window of $250 \mathrm{bp}$ (Fig. 4). The coverage of G. sibiricus transcripts suggests ample uniform coverage, with two major peaks close to genome positions $11,360,000$ and 12,360,000, which correspond to $16 \mathrm{~s}$ and $23 \mathrm{~s}$ ribosomal RNA respectively. Additionally, a table containing the transcripts which have the top BLASTX hits to Wolbachia are provided in table in an Additional file 8.

\section{Discussion}

We here present a high quality transcriptome assembly for G. sibiricus, a species of grasshopper with a large genome that is of particular evolutionary interest 

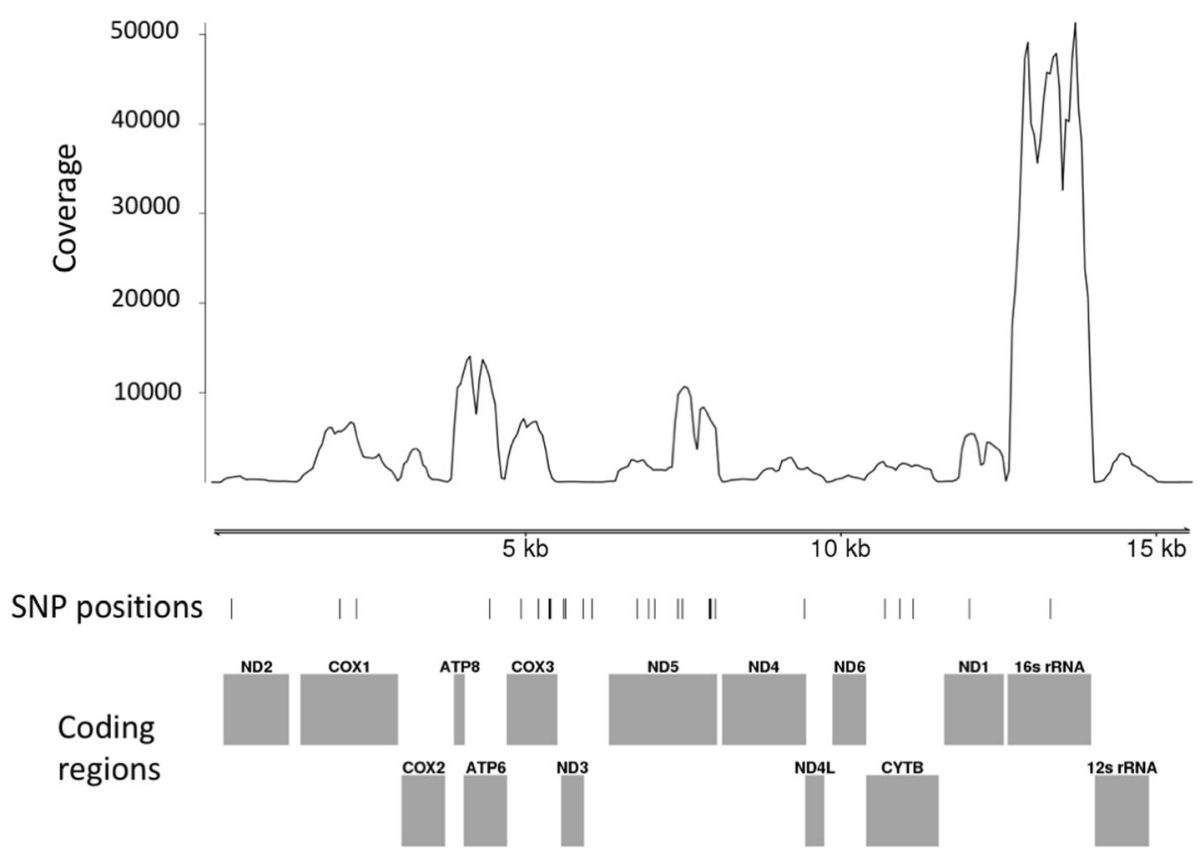

Fig. 3 Transcripts from Gomphocerus sibiricus mapping to the reference mitochondrial genome assembly. The short vertical lines indicate positions of putative SNPs. The grey shaded blocks indicate protein and rRNA coding regions

because it features a green-brown polymorphism that is shared with many other Orthopterans and seems to be maintained in natural populations by balancing selection [17]. Assembly quality statistics from BUSCO and TransRate demonstrate that the assembly is of similar quality to the only two available assemblies for the subfamily Gompocerinenae, which are also characterized by large genome sizes (the estimated genome size of G. sibiricus is approximately $8.75 \mathrm{~Gb}$ ) [25]. Our draft transcriptome provides a resource for future studies of differential gene expression, which should ultimately help to improve our understanding of the genetic basis of colour morph determination. Furthermore, our mitochondrial and Wolbachia sequence assemblies provide material for phylogenetic and comparative analyses.

Previously, a comparison of Gomphocerinae mitochondrial genomes [23] suggested that G. sibiricus is more closely related to G. licenti than to G. tibetanus, although tibietanus is sometimes listed as a subspecies of sibiricus, while licenti is considered a separate species

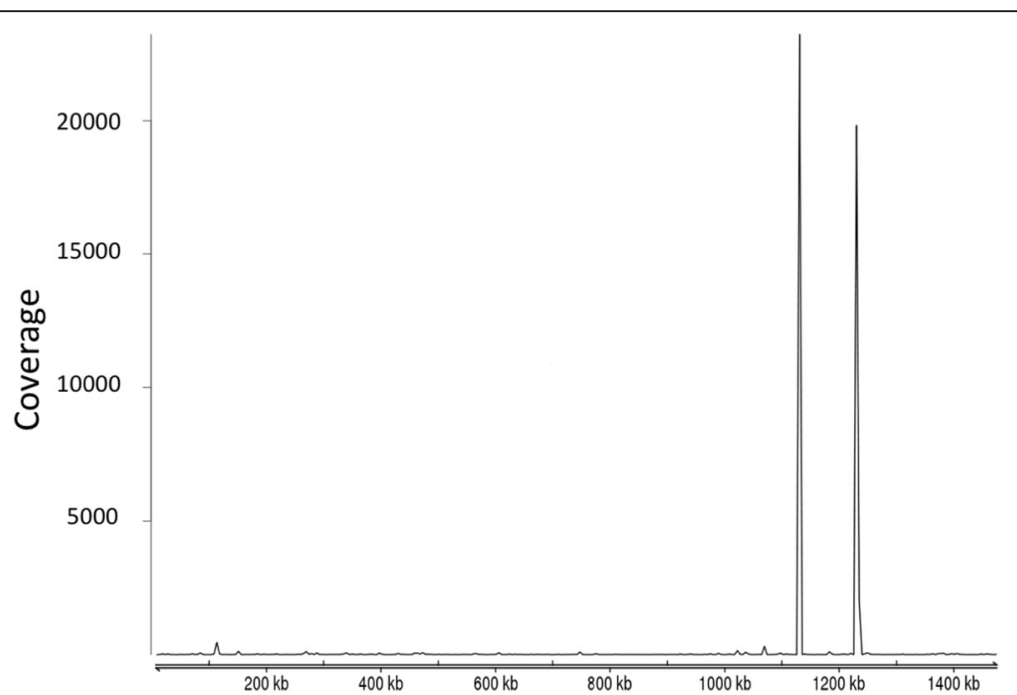

Fig. 4 Coverage of the Wolbachia genome estimated by mapping G. sibiricus transcripts against the pel wPi strain of Wolbachia. The major peaks around positons $1,136,000 \mathrm{bp}$ and 1,236,0000 bp correspond to the $16 \mathrm{~s}$ and $23 \mathrm{~s}$ rRNA coding regions 
[10]. Our analysis with an independent sample of sibiricus confirms the results of Zhang et al. [23], as we also found less divergence from licenti than from tibetanus. Despite the greater geographical distance, however, we find our sample to be closer to licenti / tibetanus than the previously published G. sibiricus mitochondrial sequence from China. This may be related to the quality of the assembly (erroneous assemblies will tend to reduce similarity) or it could potentially reflect a yet unexplored and surprising colonization history from Central Asia to the mountains in Europe.

We also found the first evidence that G. sibiricus is infected by Wolbachia, a microbial parasite that is known to manipulate host reproductive biology and can cause horizontal gene transfer [26, 27]. Our finding is not entirely unexpected given that many insect species are known to be infected by Wolbachia [28]. Moreover, Wolbachia infections have recently been reported in other grasshopper species including Chorthippus parallelus [29, 30] and Podisma saporensis [31]. Wolbachia causes cytoplasmic incompatibility in C. parallelus [30] and is suspected to play a leading role in causing hybrid dysfunction in P. saporensis [31]. Our draft transcriptome assembly therefore provides a basis for future studies investigating the evolutionary and ecological significance of Wolbachia infections in G. sibiricus.

As we were able to annotate the majority of our assembled transcripts, our assembly provides a useful tool for investigating the genetic basis of complex traits such as pigmentation. Our GO annotations allowed us to identify putative candidate genes for investigating the mechanism of colour morph determination. We demonstrated the presence of multiple transcripts that are putatively involved in pigmentation, more specifically, in the porphyrin and chlorophyll metabolism pathway (KEGG ko00860) and in haeme oxygenase 2 (biliverdin-producing, HMOX2) [32]. Furthermore, we found indirect evidence for precursors and other metabolites involved in pigmentation pathways, possibly reflecting the lack of available metabolic and biochemical resources and the significant phylogenetic distance of G. sibiricus from well-annotated model organisms. Importantly, the GO and InterPro evidence for transcripts involved in haeme and haeme-O complex metabolism (GO:0006783, GO:0006784, GO:0048034, IPR015188, IPR010961) suggests that components for a plausible mechanism to metabolize green pigments from plant sources exist in this species.

Our transcriptome assembly of G. sibiricus combines the best assembled transcripts from three different de novo transcriptome assemblers (with multiple k-mer assemblies) to detect, capture and assemble transcripts. Recently, Smith-Unna et al. [33] investigated de novo transcriptome assembly quality from 155 previously published transcriptome assemblies. They found that assemblies generated by individual assemblers yielded relatively low TransRate scores, but when multiple assemblies were combined, reduced and filtered, they yielded reasonably representative collections of sequenced read fragments. According to this particular quality metric, our draft transcriptome assembly for $G$. sibiricus lies in the approximate upper 70th percentile of the surveyed published transcriptomes, suggesting that the quality of our assembly is well above average. In our case, the combination of different assemblers was key to effective transcriptome assembly.

\section{Conclusions}

In conclusion, we generated a high quality transcriptome assembly for G. sibiricus, a species with a large genome and limited available molecular resources. Not only will our study provide a solid foundation for studying the genetic basis of green-brown colour dimorphism in $G$. sibiricus, but we also generated resources that should facilitate future studies of mitochondrial genome evolution and Wolbachia infections of Orthopterans.

\section{Methods \\ Sample collection}

Individuals of Gomphocerus sibiricus were collected from a field site at 1800-2000 m near Sierre (Valais, Switzerland) in 2013. Five individuals of their laboratory-reared offspring were selected for RNA sequencing. This included one imago brown female, one imago green female, one imago brown male, one imago green male and one last-instar green female. Sexes, colour morphs and developmental stages were mixed in order to achieve a sufficient representation of the species' transcriptome.

\section{RNA extraction, CDNA library preparation and spike-in normalisation (step 1)}

Total RNA was extracted using an innoPrep RNA kit (Analytik Jena, Jena, Germany), followed by quality control and quantification using the RNA 6000 Nano LabChip kit with the Bioanalyzer 2100 (Agilent, Santa Clara, CA, USA). The samples were pooled before cDNA library for transcriptome sequencing was constructed. In order to maximize sequencing efforts, the poly-(A)-containing mRNA was purified from total RNA using poly-(T) oligo-attached magnetic beads. This was followed by cDNA library construction, which was prepared and sequenced by the Center for Biotechnology at Bielefeld University on the Illumina MiSeq platform (San Diego, California, USA) with a maximum read length of 300. Next, an aliquot of the cDNA library was sent to a commercial facility for standard ERCC spike-in normalization, followed by an additional round of sequencing using the same protocol, to ensure that sequencing 
efforts were not only concentrated on highly abundant sequences [34]. The normalised library was used for transcriptome assembly, while the non-normalised library was used for read mapping and transcript confirmation.

\section{Pre-processing and sequence quality control and k-mer filtering (step 2)}

All pair-end reads were processed and trimmed to a maximum length of $300 \mathrm{bp}$ to remove low-quality bases. The resulting reads were assessed for quality using FastQC (version 0.11.15). Sequence quality and adapter trimming was performed using the trimmomatic tool (version 0.36) [35], with a four base sliding window. Bases below a phred quality score of 15 were removed as were reads with a sequence length below 150 using the setting '2:30:10 LEADING:3 TRAILING:3 SLIDINGWINDOW:4:15 MINLEN:150'. Furthermore, the reads were in silico normalized using Khmer (version 2.0) following the authors' recommended protocol for trimming reads with variable coverage (recipe 7) [36]. Additional artefact filtering was carried out using the FASTX toolkit (version 0.0.14). This resulted in a final read dataset of $5,070,036$ paired-end reads with a median read length of $249 \mathrm{bp}$ for the normalised read dataset.

\section{Transcriptome assemblies (step 3)}

We assembled the G. sibiricus transcriptome using the de novo transcriptome assembly packages SOAPdenovotrans [37], Trinity [38] and Oases-Velvet [39]. An overview of this process is given in Fig. 5. First, we used SOAPdenovo-trans (version 1.03) to build 54 de novo assemblies with k-mer values of 21 to 127 (in steps of 2) with an average insert size of $475 \mathrm{bp}$, using -F and -L 300 command parameters to set filling the gaps in scaffolds and shortest contig length for scaffolding to $300 \mathrm{bp}$. Second, we used Trinity (release v2.2.0) with the default settings, the minimum transcript length set to $300 \mathrm{bp}$, and with in-silico read normalization with default settings. Third, Oases-Velvet (version 0.2.09) was used to build eighteen de novo assemblies with k-mer values of 21 to 55 (in steps of 2) with an insert length of $475 \mathrm{bp}$ and the minimum transcript length set to $300 \mathrm{bp}$. Assemblies with higher k-mer values were not possible due to memory constraints.

\section{Multi-assembly merging and duplicate contig reduction (step 4)}

All of the assemblies were collated into a single file and followed by removal of duplicate and redundant contigs. CD-HIT (cd-hit-est, version 4.6) [40], a greedy incremental clustering algorithm, was used to remove duplicate and highly similar sequences (sequence similarity greater than $90 \%$ identity) to generate representative sequences of all of the assembled transcripts from all of the assemblies. This approach allowed us to capture as many unique transcripts as possible from three different de novo transcriptome assemblers.

\section{Transcript mapping and quantification (step 5)}

The quality filtered reads were mapped to our de novo transcriptome assembly and the number of mapping reads per transcript was quantified using

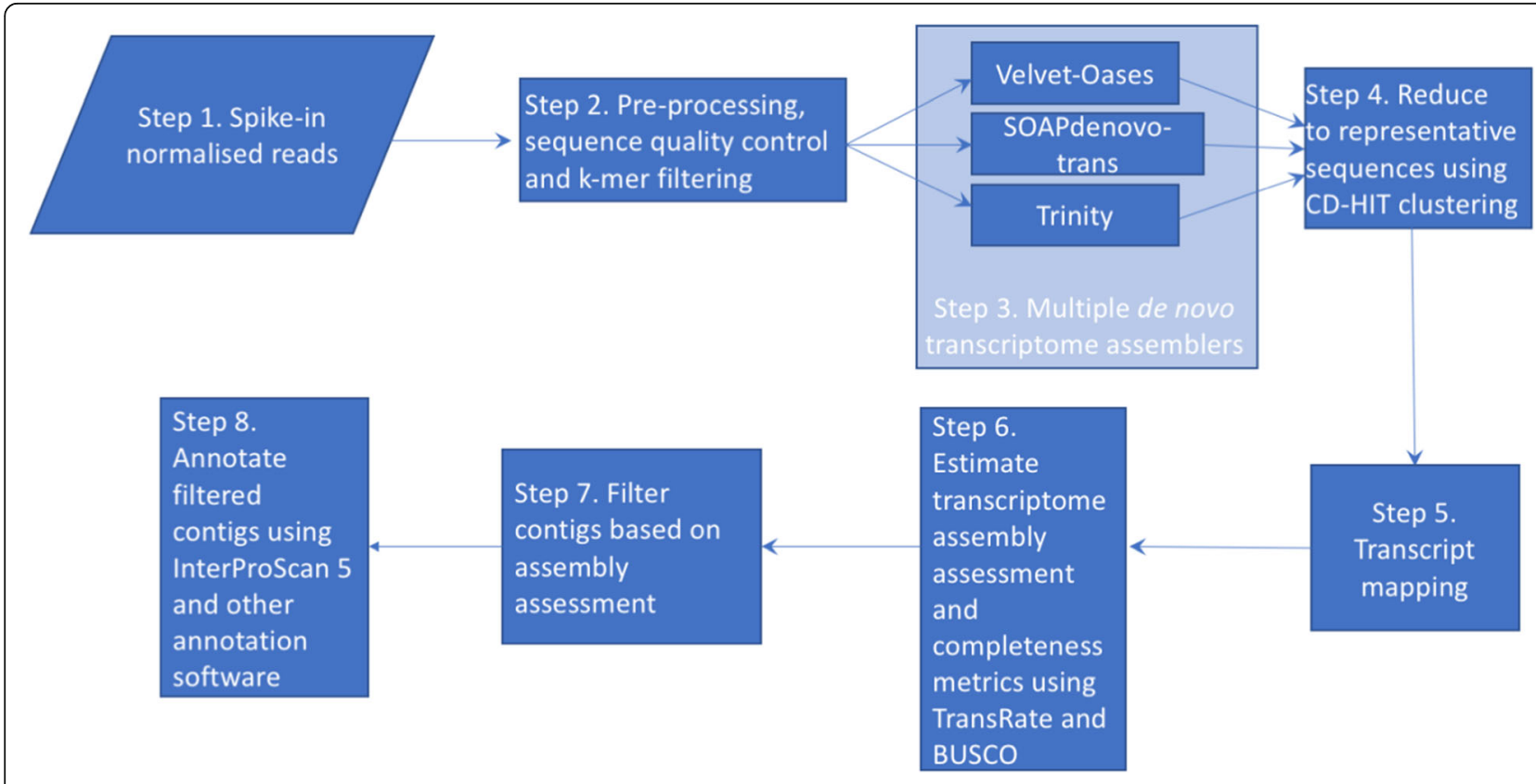

Fig. 5 Flow chart showing a summary of the de novo assembly procedure and downstream analyses 
BWA (version 0.712-r1039) [41]. Per contig and per base coverage was calculated using BBMap [42] and SAMtools (version 1.4) [43].

\section{Assessment of assembled transcriptome and contig filtering (step 6 and 7)}

We assessed the quality of the de novo assembly using the TransRate package (version 1.0.3) [33]. This assesses transcriptome assembly accuracy using read and contig data and estimates individual contig and overall assembly quality. Contigs that scored low on the contig assessment criteria (contig score components: $\mathrm{S}\left(\mathrm{C}_{\text {seg }}\right)$ and $\mathrm{S}\left(\mathrm{C}_{\text {cov }}\right)$, with default thresholds as suggested by TransRate), were filtered out. We then compared our empirical assembly metrics to the published transcriptomes of two other Gomphocerine species for which transcriptome assemblies have been published (Stenobothrus lineatus [13] and Chorthippus biguttulus [44]). Assembly completeness was assessed with Benchmarking Universal Single-Copy Orthologs (BUSCO, version 2.0) [45]. BUSCO defines a set of core genes for a given group or lineage and uses these genes as proxy for minimum completeness assuming that these genes should encode a large set of core genes. For this analysis, we used the genome and transcriptome completeness assessment tool in transcriptome assessment mode with the insect lineage database (insecta_orthoDB9, created 13/02/ 2016).

\section{Annotation and sequence analysis (step 8)}

After assembly and assembly assessment, we annotated and analysed the assembly. InterProScan 5 is a widely used sequence analysis framework to search for various analytical signatures from different databases including the InterPro protein database. The draft transcript sequence signatures were scanned against InterPro's signatures (version 5.22) [46]. TMHMM, SignalP, TIGRFAMs, Prosite, Panther, PFam, PIRSF, CDD, COILS, and Gene3D applications were selected to run with the transcripts using the InterScanPro 5 framework. Additional annotation was generated using the dammit! annotation pipeline (https:/github.com/dib-lab/dammit/). This pipeline was set to use the Pfam, Rfam, OrthoDB, uniref90 and BUSCO arthropoda databases in the '--full' mode. All assembled contigs were also searched against the BLASTx non-redundant protein database $(\mathrm{Nr})$ and results with expect values (e-values) below $10^{-6}$ were reported. To visualize the BLAST profile of the assembly, we selected the best matches (based on best bit-scores) from every transcript and plotted the counts of taxonomic classes found.
SNP calling and estimation of minor allele frequencies We detected SNPs from the transcriptome by mapping the quality-filtered reads to the final set of assembled transcripts using the $B W A$ aligner (version 0.712-r1039) [47] (using default settings) followed by variant detection with VarScan (version 2.4.2) [48] with minimum coverage set to 30 , a minimum expected variant frequency set to 0.1 , and $p$-value threshold of 0.01 . In order to visualize our variants with respect to sequencing depth, we estimated the two-dimensional kernel density of the minor allele frequency and plotted this as a contour plot.

Searches for candidate green-brown pigmentation genes Gene ontology terms (GO terms) associated with pigmentation (GO:0043473) and haeme-metabolism (GO:0004392, GO:0006784 and GO:0048034) were selected as possible candidates for genes associated with the observed green-brown dimorphism. Furthermore, InterPro and KEGG terms (IPR010961, IPR015118, IPR002051, KEGG00860 + 1.14.14.18) associated with haeme oxygenase (the pathway leading to biliverdin production) were also added to the annotation search. The presence of these GO terms illustrates that putative candidates for pigmentation are represented in our assembly.

\section{Analysis of the mitochondrial genome}

We also assembled the mitochondrial transcriptome of G. sibiricus. Reads were mapped to a the reference mitochondrial genome from a specimen of the same species from China (NCBI Reference Sequence NC_021103.1) [23]. This was done using $B W A$ with default settings, and coverage was estimated using BBMap [42] and SAMtools (version 1.4) [43]. Furthermore, the assembled transcripts were BLASTed against the reference mitochondrial genome to screen for potential chimeric components in the assembled transcripts. Variant calling was again performed using VarScan (version 2.4.2) [48] and all of the results were plotted and annotated using the Gviz package [49] on Bioconductor [50].

Finally, we aligned and estimated the sequence distance of four closely related Gomphocerinae mitogenomes, namely G. rufus (RefSeq: NC_014349), G. licenti (RefSeq: NC_013847), G. tibetanus (RefSeq: NC_015478), G. sibiricus from the Tianshan Mountians, Xinjiang, China (RefSeq: NC_021103) and our data from G. sibiricus from the European Alps. The multiple sequence alignment was estimated using MAFFT [51] with the default setting in 'auto' mode. The program dnadist from the PHYLIP package (version 3.696) [52] was used with default settings (F84 substitution model, transition/transversion ratio 2.0, using empirical base frequencies) to estimate pairwise sequence distances. The program dnaml from the PHYLIP package [52] was used to estimate the best fitting phylogenetic tree with default settings (transition/transversion 
ratio 2.0, constant rate variation among sites), with randomized input order of sequences, and G. rufus set as the outgroup and plotted (see Additional file 4: Figure S4).

\section{Additional files}

Additional file 1: Figure S1. Log of average fold coverage versus log of
contig length. (DOCX $58 \mathrm{~kb}$ ) Additional file 2: Figure S2. Top 20 taxa classes reported by BLASTX. (DOCX $50 \mathrm{~kb}$ )

Additional file 3: Figure S3. Classifications of GO terms of contigs. (DOCX $251 \mathrm{~kb}$ )

Additional file 4: Figure S4. A phylogeny based on mitochondrial sequences of four Gomphocerine grasshopper species. (JPG $35 \mathrm{~kb}$ )

Additional file 5: Table S1. A Summary table of the annotation of the contigs using the dammit! Pipeline. (DOCX $13 \mathrm{~kb}$ )

Additional file 6: Table S2. Sequence divergence matrix from four Gomphocerine grasshopper species. (DOCX $13 \mathrm{~kb}$ )

Additional file 7: Table S3. Read mapping statistics for non-rRNA reads. (DOCX $12 \mathrm{~kb})$

Additional file 8: Table of top Wolbachia BLASTX hits from the assembly. (CSV $104 \mathrm{~kb})$

\section{Abbreviations}

BLAST: Basic local alignment tool; bp: base pair; BUSCO: Benchmarking Universal Single-Copy Orthologs; ERCC: External RNA Control Consortium; GO: Gene Ontology; KEGG: Kyoto Encyclopedia of Genes and Genomes; MAF: Minor Allele Frequency; ORF: Open Reading Frames

\section{Acknowledgements}

We are grateful to Amy R. Backhouse for carrying out the sample preparations and RNA extractions and Anika Winkler for further lab assistance. We would also like to thank Drs. Emily Humble and Luke Eberhart-Philips for assisting us in plotting data. We also thank The Center for Biotechnology (CeBiTec) at Bielefeld University to provide compute cluster resources to facilitate this endeavour.

\section{Funding}

This study was supported by an Emmy-Noether fellowship by the German Research Foundation (DFG; SCHI 1188/1-1). The funding body played no role in the design of the study, collection of samples, analysis and interpretation of data, and writing the manuscript.

\section{Availability of data and materials}

The draft transcriptome and read data are deposited in the Transcriptome Shotgun Assembly Sequence database and in the Short Read Archive respectively, under BioProject ID:PRJNA525981 with the accession id: GHKV00000000,

\section{Authors' contributions}

AS, $\mathrm{HS}$ and $\mathrm{JIH}$, conceived and designed the project. AS executed the bioinformatic pipelines and performed the data analysis. AS, JIH and HS drafted the manuscript. All authors contributed to revision and approved the final version

\section{Ethics approval and consent to participate}

The authors declare that the experiments comply with the current law of the country in which they had been performed. All sampling was carried out with non-protected species in non-protected areas.

\section{Consent for publication}

Not applicable.

\section{Competing interests}

The authors declare that they have no competing interests.

\section{Publisher's Note}

Springer Nature remains neutral with regard to jurisdictional claims in published maps and institutional affiliations.

Received: 30 May 2018 Accepted: 30 April 2019

Published online: 14 May 2019

\section{References}

1. Lee YW, Gould BA, Stinchcombe JR. Identifying the genes underlying quantitative traits: a rationale for the QTN programme. Aob Plants. 2014;6: plu004.

2. Schielzeth $H$, Husby A. Challenges and prospects in genome-wide QTL mapping of standing genetic variation in natural populations. Ann N Y Acad Sci. 2014;1320:35-57.

3. Schatz MC, Delcher AL, Salzberg SL. Assembly of large genomes using second-generation sequencing. Genome Res. 2010;20(9):1165-73.

4. Nowoshilow S, Schloissnig S, Fei JF, Dahl A, Pang AWC, Pippel M, Winkler S, Hastie AR, Young G, Roscito JG, et al. The axolotl genome and the evolution of key tissue formation regulators. Nature. 2018;554(7690):50-5.

5. Martin JA, Wang Z. Next-generation transcriptome assembly. Nat Rev Genet. 2011;12(10):671-82.

6. Parmigiani G, Garrett ES, Irizarry RA. Zeger SL (eds.): the analysis of gene expression data : methods and software. New York: Springer; 2003.

7. Wang Z, Gerstein M, Snyder M. RNA-Seq: a revolutionary tool for transcriptomics. Nat Rev Genet. 2009;10(1):57-63.

8. Zhang R, Calixto Cristiane PG, Marquez Y, Venhuizen P, Tzioutziou NA, Guo W, Spensley M, Entizne JC, Lewandowska D, ten Have S, et al. A high quality Arabidopsis transcriptome for accurate transcript-level analysis of alternative splicing. Nucleic Acids Res. 2017;45(9):5061-73.

9. Gregory TR. Animal Genome Size Database; 2018.

10. Cigliano MM, Braun H, Eades DC, Otte D. Orthoptera Species File (Version 5. 0/5.0). In. 2018.

11. Badisco L, Huybrechts J, Simonet G, Verlinden H, Marchal E, Huybrechts R, Schoofs L, De Loof A, Vanden Broeck J. Transcriptome analysis of the desert locust central nervous system: production and annotation of a Schistocerca gregaria EST database. PLoS One. 2011;6(3):e17274.

12. Wang $X H$, Fang $X D$, Yang $P C$, Jiang $X T$, Jiang $F$, Zhao DJ, Li BL, Cui F, Wei $\mathrm{JN}, \mathrm{Ma} C \mathrm{~A}$, et al. The locust genome provides insight into swarm formation and long-distance flight. Nat Commun. 2014;5:2957.

13. Misof B, Liu S, Meusemann K, Peters RS, Donath A, Mayer C, Frandsen PB, Ware J, Flouri T, Beutel RG, et al. Phylogenomics resolves the timing and pattern of insect evolution. Science. 2014;346(6210):763-7.

14. Berdan EL, Finck J, Johnston PR, Waurick I, Mazzoni CJ, Mayer F. Transcriptome profiling of ontogeny in the acridid grasshopper Chorthippus biguttulus. PLoS One. 2017;12(5):e0177367.

15. Song H. Density-dependent phase polyphenism in nonmodel locusts: a minireview. Psyche. 2011;2011 741769.

16. Bellmann $\mathrm{H}$, Luquet $\mathrm{CH}$. Guide des sauterelles, grillons et criquets d'Europe occidentale. Paris: Delachaux et Niestlé; 2009.

17. Dieker P, Beckmann L, Teckentrup J, Schielzeth H. Spatial analyses of two colour polymorphisms in an alpine grasshopper reveal a role of small-scale heterogeneity. Ecol Evol. 2018. https://doi.org/10.1002/ece1003.4156.

18. Valverde JP, Eggert H, Kurtz J, Schielzeth H. Condition-dependence and sexual ornamentation: effects of immune challenges on a highly sexually dimorphic grasshopper. Insect Sci. 2017. https://doi.org/10.1111/1744-7917. 12448.

19. Valverde JP, Schielzeth $\mathrm{H}$. What triggers colour change? Background colour and temperature effects on the development of an alpine grasshopper. BMC Evol Biol. 2015:15:168

20. Fuzeau-Braesch S. Pigments and color changes. Annu Rev Entomol. 1972;17: 403-24.

21. Cerveau N, Jackson DJ. Combining independent de novo assemblies optimizes the coding transcriptome for nonconventional model eukaryotic organisms. BMC Bioinformatics. 2016;17(1):525.

22. Haznedaroglu BZ, Reeves D, Rismani-Yazdi H, Peccia J. Optimization of de novo transcriptome assembly from high-throughput short read sequencing data improves functional annotation for non-model organisms. BMC Bioinformatics. 2012;13(1):170.

23. Zhang H-L, Zhao L, Zheng Z-M, Huang Y. Complete mitochondrial genome of Gomphocerus sibiricus (Orthoptera: Acrididae) and comparative analysis in four Gomphocerinae mitogenomes. Zool Sci. 2013;30(3):192-204. 
24. Klasson L, Walker T, Sebaihia M, Sanders MJ, Quail MA, Lord A, Sanders S, Earl J, O'Neill SL, Thomson N, et al. Genome evolution of Wolbachia strain wPip from the Culex pipiens group. Mol Biol Evol. 2008;25(9):1877-87.

25. Gosalvez J, López-Fernandez C, Esponda P. Variability of the DNA content in five Orthopteran species. Caryologia. 1980;33(2):275-81.

26. Ahmed MZ, Breinholt JW, Kawahara AY: Evidence for common horizontal transmission of Wolbachia among butterflies and moths. BMC Evol Biol 2016, 16(1):118-118.

27. Werren JH, Baldo L, Clark ME. Wolbachia: master manipulators of invertebrate biology. Nat Rev Microbiol. 2008;6(10):741-51.

28. Hilgenboecker K, Hammerstein P, Schlattmann P, Telschow A, Werren JH. How many species are infected with Wolbachia? - a statistical analysis of current data. FEMS Microbiol Lett. 2008;281(2):215-20.

29. Zabal-Aguirre M, Arroyo F, Bella JL. Distribution of Wolbachia infection in Chorthippus parallelus populations within and beyond a Pyrenean hybrid zone. Heredity. 2009;104:174.

30. Zabal-Aguirre M, Arroyo F, García-Hurtado J, Torre J, Hewitt GM, Bella JL. Wolbachia effects in natural populations of Chorthippus parallelus from the Pyrenean hybrid zone. J Evol Biol. 2014;27(6):1136-48.

31. Bugrov AG, Ilinsky YY, Strunov A, Zhukova M, Kiseleva E, Si A, Tatsuta H. First evidence of Wolbachia infection in populations of grasshopper Podisma sapporensis (Orthoptera: Acrididae). Entomological Science. 2016;19(3):296300.

32. Ishikawa K, Takeuchi N, Takahashi S, Matera KM, Sato M, Shibahara S, Rousseau DL, Ikeda-Saito M, Yoshida T. Heme Oxygenase-2: properties of the heme complex of the purified tryptic fragment of recombinant human heme oxygenase-2. J Biol Chem. 1995;270(11):6345-50.

33. Smith-Unna R, Boursnell C, Patro R, Hibberd JM, Kelly S. TransRate: reference-free quality assessment of de novo transcriptome assemblies. Genome Res. 2016;26(8):1134-44.

34. Jiang L, Schlesinger F, Davis CA, Zhang Y, Li R, Salit M, Gingeras TR, Oliver B. Synthetic spike-in standards for RNA-seq experiments. Genome Res. 2011; 21(9):1543-51

35. Bolger AM, Lohse M, Usadel B. Trimmomatic: a flexible trimmer for Illumina sequence data. Bioinformatics. 2014;30(15):2114-20.

36. Crusoe MR, Alameldin HF, Awad S, Boucher E, Caldwell A, Cartwright R, Charbonneau A, Constantinides B, Edvenson G, Fay S, et al. The khmer software package: enabling efficient nucleotide sequence analysis. F1000Research. 2015:4:900

37. Xie Y, Wu G, Tang J, Luo R, Patterson J, Liu S, Huang W, He G, Gu S, Li S, et al. SOAPdenovo-trans: de novo transcriptome assembly with short RNA-Seq reads. Bioinformatics. 2014;30(12):1660-6.

38. Haas BJ, Papanicolaou A, Yassour M, Grabherr M, Blood PD, Bowden J, Couger MB, Eccles D, Li B, Lieber M, et al. De novo transcript sequence reconstruction from RNA-seq using the trinity platform for reference generation and analysis. Nat Protoc. 2013;8(8):1494-512.

39. Schulz MH, Zerbino DR, Vingron M, Birney E. Oases: robust de novo RNAseq assembly across the dynamic range of expression levels. Bioinformatics. 2012;28(8):1086-92.

40. Li WZ, Godzik A. Cd-hit: a fast program for clustering and comparing large sets of protein or nucleotide sequences. Bioinformatics. 2006:22(13):1658-9.

41. Li H: Aligning sequence reads, clone sequences and assembly contigs with BWA-MEM. arXive preprint 2013:available at https://arxiv.org/abs/1303.3997.

42. Bushnell B. BBMap short read aligner. In: University of California, Berkeley, California; 2016

43. Li H, Handsaker B, Wysoker A, Fennell T, Ruan J, Homer N, Marth G, Abecasis G, Durbin R. Genome project data processing S: the sequence alignment/ map format and SAMtools. Bioinformatics. 2009;25(16):2078-9.

44. Berdan EL, Mazzoni CJ, Waurick I, Roehr JT, Mayer F. A population genomic scan in Chorthippus grasshoppers unveils previously unknown phenotypic divergence. Mol Ecol. 2015;24(15):3918-30.

45. Simão FA, Waterhouse RM, loannidis P, Kriventseva EV, Zdobnov EM BUSCO: assessing genome assembly and annotation completeness with single-copy orthologs. Bioinformatics. 2015:31(19):3210-2.

46. Jones P, Binns D, Chang HY, Fraser M, Li WZ, McAnulla C, McWilliam H, Maslen J, Mitchell A, Nuka G, et al. InterProScan 5: genome-scale protein function classification. Bioinformatics. 2014;30(9):1236-40.

47. Li H, Durbin R. Fast and accurate short read alignment with burrowswheeler transform. Bioinformatics. 2009;25(14):1754-60.

48. Koboldt DC, Chen K, Wylie T, Larson DE, McLellan MD, Mardis ER, Weinstock GM, Wilson RK, Ding L. VarScan: variant detection in massively parallel sequencing of individual and pooled samples. Bioinformatics. 2009;25(17): 2283-5.

49. Hahne F, Ivanek R. Visualizing genomic data using Gviz and bioconductor In: Mathé E, Davis S, editors. Statistical genomics: methods and protocols. New York, NY: Springer New York; 2016. p. 335-51.

50. Huber W, Carey VJ, Gentleman R, Anders S, Carlson M, Carvalho BS, Bravo HC, Davis S, Gatto L, Girke T, et al. Orchestrating high-throughput genomic analysis with bioconductor. Nat Methods. 2015;12(2):115-21.

51. Katoh K, Standley DM. MAFFT multiple sequence alignment software version 7: improvements in performance and usability. Mol Biol Evol. 2013; 30(4):772-80.

52. Felsenstein J. Comparative methods with sampling error and within-species variation: contrasts revisited and revised. Am Nat. 2008:171(6):713-25.
Ready to submit your research? Choose BMC and benefit from:

- fast, convenient online submission

- thorough peer review by experienced researchers in your field

- rapid publication on acceptance

- support for research data, including large and complex data types

- gold Open Access which fosters wider collaboration and increased citations

- maximum visibility for your research: over $100 \mathrm{M}$ website views per year

At $\mathrm{BMC}$, research is always in progress.

Learn more biomedcentral.com/submissions 